\title{
Resonant Converter Fed PMDC Motor Drive for Low Voltage Applications
}

\author{
Vaisakh. T \\ Post Graduate, Power Electronics and Drives
}

\begin{abstract}
At zero stator frequency the stator quantities are not affected by the rotor speed and, therefore, the speed cannot be observed from measured stator voltages and currents. Further, the stator voltage provided by the inverter is difficult to measure and sometimes is assumed to be equal to the reference voltage or calculated from the DC link voltage and inverter switches conditions. The errors caused are often negligible in high speeds. However, they can be relatively high in low speeds, since the voltages are reduced to maintain the reference flux and the errors due to the voltage drops and dead times in the switching devices become more prominent in lower output voltage range. However, they need a high computational effort to be implemented and a kind of intervention in the machine is necessary Despite the many attempts to obtain accurate flux and speed estimation for wide speed range induction motor drives proposed recently, it seems that the solutions do not meet simultaneously the simplicity, accuracy and reliability requisites to be largely accepted and used in the industries. This instantaneous average voltage-measuring scheme was used to obtain the results in this paper. The scheme is simple and inexpensive.
\end{abstract}

Keywords: Soft switching DC-DC converter, Zero Current Switching, PI controller. PMDC motor

\section{Introduction}

It is very important to control the speed of induction motors in industrial and engineering applications. Efficient control strategies are used for reducing operation cost too. Speed control techniques of induction motors can be broadly classified into two types - scalar control and vector control. Scalar control involves controlling the magnitude of voltage or frequency of the induction motor. Simple converter structure, motor-friendly waveforms, inherent four-quadrant operation capability and reliable short-circuit protection are features that make current-source inverter (CSI) well suited for medium-voltage drives applications. By adopting IGBT can achieve improved line/load current harmonics, superior power factor, and reduced costs with the possibility of eliminating the input transformer. Field-oriented control strategies are widely employed in the high-power currentsource drives to improve system dynamics and reliability. Zero-speed operation plays an important role in applications such as cranes, hoists, and traction drives, where maintaining the desired torque down to zero speed or starting the load with a high torque from zero speed is highly desirable. The most popular induction motor speed control methods are based on frequency converters. They contain rectifier and inverter. A very popular present time control methods is known as Field Orientated Control (FOC). In this method the motor equation are transformed in a coordinate system that rotates with the rotor flux vector. The method allows control Voltage source inverter fed induction motor drive not only amplitude and frequency, like in Voltage/Frequency control, but also the phase of the voltage, current and flux vectors. The drive is controlled with rotor flux field orientation, where the flux is identified by current model. Filter capacitors are connected at the output of the CSI to assist with current commutation and harmonics filtering. Thus, the inverter $d, q$-axis currents are different from the stator $d, q$-axis currents. The impact of the capacitors on the drive dynamic performance is systematically analyzed.

\section{Block Diagram}

Single phase supply of 230 Volts AC Voltage is given to the Diode Bridge Rectifier IC of main circuit. The Diode bridge rectifier IC converts AC voltage to pulsating $\mathrm{DC}$ voltage. The pulsating DC voltage is given to Current source inverter as input. The Voltage source inverter converts pulsating DC voltage into AC voltage. Speed measurement is done by the speed sensor and it is given to the dsPIC microcontroller. It acts as the feedback loop and also generates the gate pulse depends on the speed. Speed control is done by PWM technique which is fed as a program in the dsPIC microcontroller. The output of the controller generates the PWM signal which is given to the inverter as gate signal and whose control and modulation of frequency is extremely high to avoid the ripple caused by the small inductance winding's modulation. Driver circuit which consists of Photocoupler needed to isolate the low voltage dsPIC Microcontroller unit from the high voltage main circuit.

\section{Link}

A DC link exists between a rectifier and an inverter. The DC link capacitor helps to keep these transients from radiating back to the input. This can also help prevent the switching network from oscillating or triggering inadvertently at an inappropriate moment and causing a short. Additionally, if the input is not multiple-phase, the capacitor helps provide a source of energy when the input waveform is near zero. 


\section{International Journal of Science and Research (IJSR) \\ ISSN (Online): 2319-7064}

Index Copernicus Value (2013): 6.14 | Impact Factor (2014): 5.611

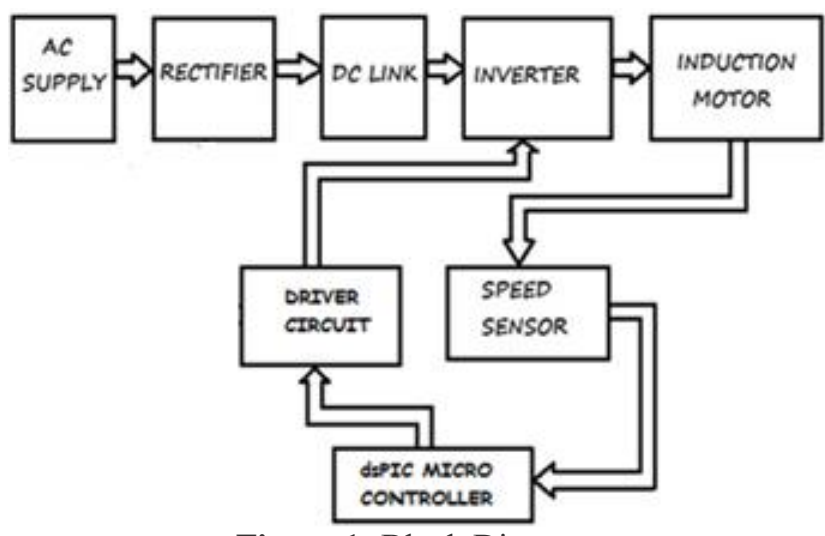

Figure 1: Block Diagram

\section{Inverter}

An inverter is an electrical device that converts direct current (DC) to alternating current (AC). The converted AC can be at any required voltage and frequency with the use of appropriate transformers, switching, and control circuits. Solid-state inverters have no moving parts and are used in a wide range of applications, from small switching power supplies in computers, to large electric utility high-voltage

direct current applications that transport bulk power. Inverters are commonly used to supply AC power from DC sources such as solar panels or batteries.

\section{Microcontroller Unit}

The dsPIC30F2010 devices contain extensive Digital Signal Processor (DSP) functionality within high-performance 16bitmicrocontroller architecture and 28 pin. The dsPIC30F2010 devices contain internal program Flash memory for executing user code.

\section{Opto Coupler}

Opto-isolators prevent high voltages from affecting the system receiving the signal. Commercially available optoisolators withstand input-to-output voltages up to $10 \mathrm{kV}$ and voltage transients with speeds up to $10 \mathrm{kV} / \underline{\mu \mathrm{s}}$. A common type of opto-isolator consists of an LED and a phototransistor in the same package.

\section{Circuit Diagram}

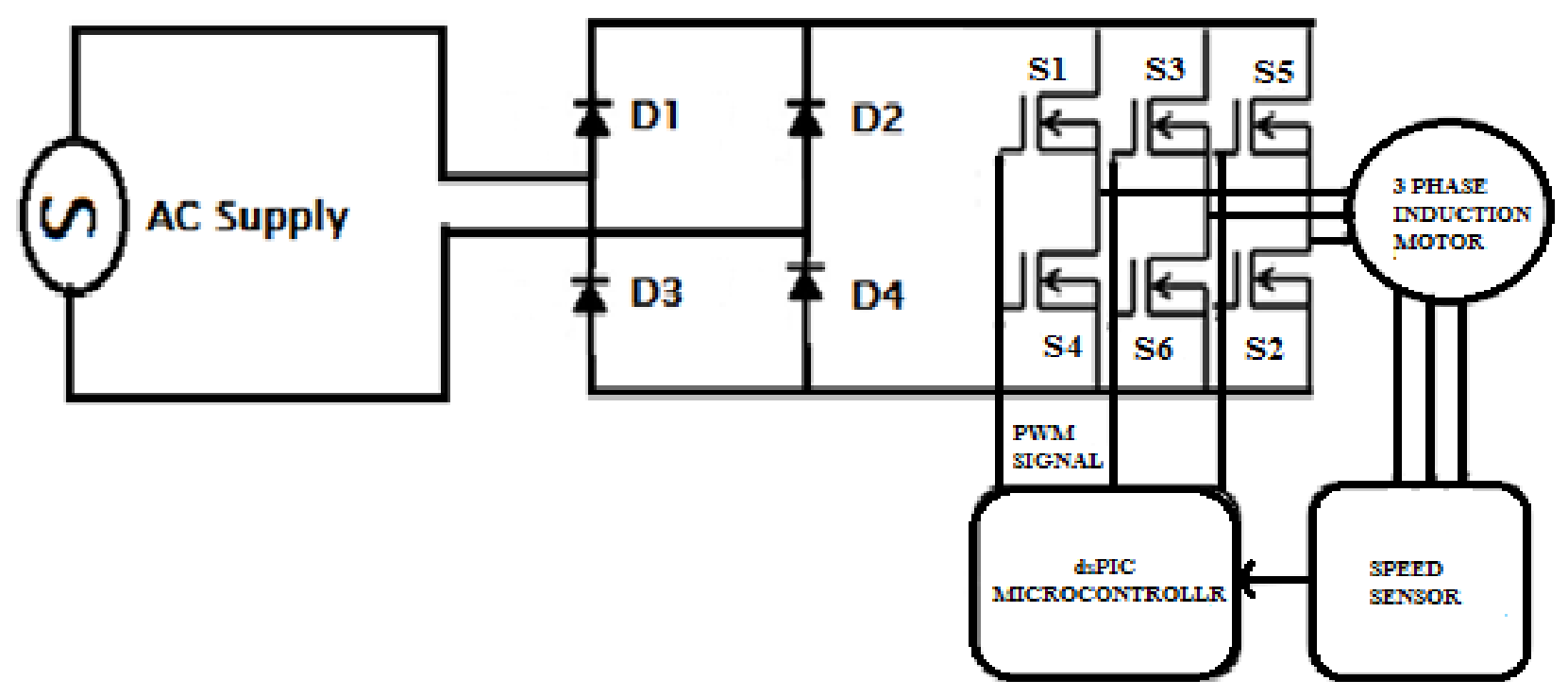

Figure 2: Circuit Diagram

\section{Circuit Digram Description}

CSI-fed motor drives have filter capacitors connected at the output of the inverter. This means that a portion of the inverter currents go through the capacitors. The influence of the filter capacitors on the system control is investigated in this section.

\section{Current Flux Model}

The current model is utilized for the flux estimation, where the rotor flux components can be synthesized easily with the help of speed and current signals. The relationship among

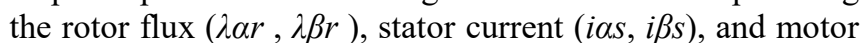
speed $(\omega r)$ in two-phase stationary reference frame is shown.

$$
\left(p+\frac{1}{\tau_{r}}\right) \lambda_{\alpha r}=\frac{L_{m}}{\tau_{r}} i_{\alpha s}-\omega_{r} \lambda_{\beta r}
$$

$$
\left(p+\frac{1}{\tau_{r}}\right) \lambda_{\beta s}=\frac{L_{m}}{\tau_{r}} i_{\beta s}+\omega_{r \lambda_{\alpha r}}
$$

Where $\tau r=L r / R r$ is the rotor circuit time constant, and $p$ is the derivative operator.

\section{Coupling Analysis of Stator D, Q-AXIS Currents}

The inverter reference currents can be expressed as follows

$$
\begin{aligned}
& i^{*}{ }_{d \omega}=i_{c d}+i^{*}{ }_{d s} \\
& i^{*}{ }_{q \omega}=i_{c q}+i{ }^{*}{ }_{q s}
\end{aligned}
$$

icd and icq are the estimated capacitor d, q-axis currents. To reduce the sensitivity and noise caused by the derivative terms, the estimated capacitor currents are usually simplified as follows 


\section{International Journal of Science and Research (IJSR) \\ ISSN (Online): 2319-7064}

Index Copernicus Value (2013): 6.14 | Impact Factor (2014): 5.611

$i_{c d}=-\omega_{e} v_{q s} C_{f}$

$i_{c q}=\omega_{e} v_{d s} C_{f}$

Where $\mathrm{Cf}$, $\omega \mathrm{e}$, vds, and vqs are the inverter-side filter capacitance, motor electrical angular frequency, and stator $\mathrm{d}$-axis and q-axis voltages, respectively.

$$
\left(\begin{array}{c}
v_{d s} \\
v_{q s}
\end{array}\right)=\left(\begin{array}{cc}
s \sigma L_{s}+R_{s} & -\omega_{e} \sigma L_{s} \\
\omega_{e} \sigma L_{s} & s \sigma L_{s}+R_{s}
\end{array}\right)\left(\begin{array}{l}
i_{d s} \\
i_{q s}
\end{array}\right)+\left(\begin{array}{c}
e_{d} \\
e_{q}
\end{array}\right)
$$

Where $\sigma$, ed, and eq are the induction motor total leakage factor $(1-\mathrm{L} 2 \mathrm{~m} /(\mathrm{LsLr}))$, and $\mathrm{d}$ - and q-axis back electromotive force (EMF), respectively. Under rotor flux orientation, the rotor flux vector is aligned with the d-axis and its amplitude is kept constant. Thus, the induction motor back EMF can be obtained as follows:

$$
\begin{aligned}
& e_{d}=0 \\
& e_{q}=\frac{\omega_{e \lambda_{d r}} L_{m}}{L_{r}}
\end{aligned}
$$

The rotor flux controller and speed controller provide the motor d, q-axis reference currents $\mathrm{i} * \mathrm{ds}$ and $\mathrm{i} * \mathrm{qs}$, respectively. After the capacitor current compensation, the inverter reference currents $i * d w$ and $i * q w$ are generated. Subsequently, the inverter real currents idw and iqw can be obtained with an inverter processing delay of Tdi and then supply the filter capacitor and motor.

\section{Resultant Input Waveform}

The Figure.4.shows the resultant input waveform of d-axis. With 1 p.u. step torque variation, the system performance without and with the load torque observer are show in Figure.4.Without the load torque observer, the DC link current and stator $d$-axis currents exhibit almost no transient oscillations due to comparatively slow speed response. The speed drop is up to $28 \mathrm{r} / \mathrm{min}$. With the help of load torque observer, the speed drop is reduced to $14 \mathrm{r} / \mathrm{min}$, while the DC link current and stator $d$-axis currents suffer about two cycles of transient resonance.The Figure.5 shows the resultant input waveform of q-axis. With 1 p.u. step torque variation, the system performance without and with the load torque observer are show in Figure.5. Without the load torque observer, the DC link current and stator $q$-axis currents exhibit almost no transient oscillations due to comparatively slow speed response. The speed drop is up to $28 \mathrm{r} / \mathrm{min}$. With the help of load torque observer, the speed drop is reduced to $14 \mathrm{r} / \mathrm{min}$, while the DC link current and stator $q$-axis currents suffer about two cycles of transient resonance

\section{MATLAB/Simulink Model}

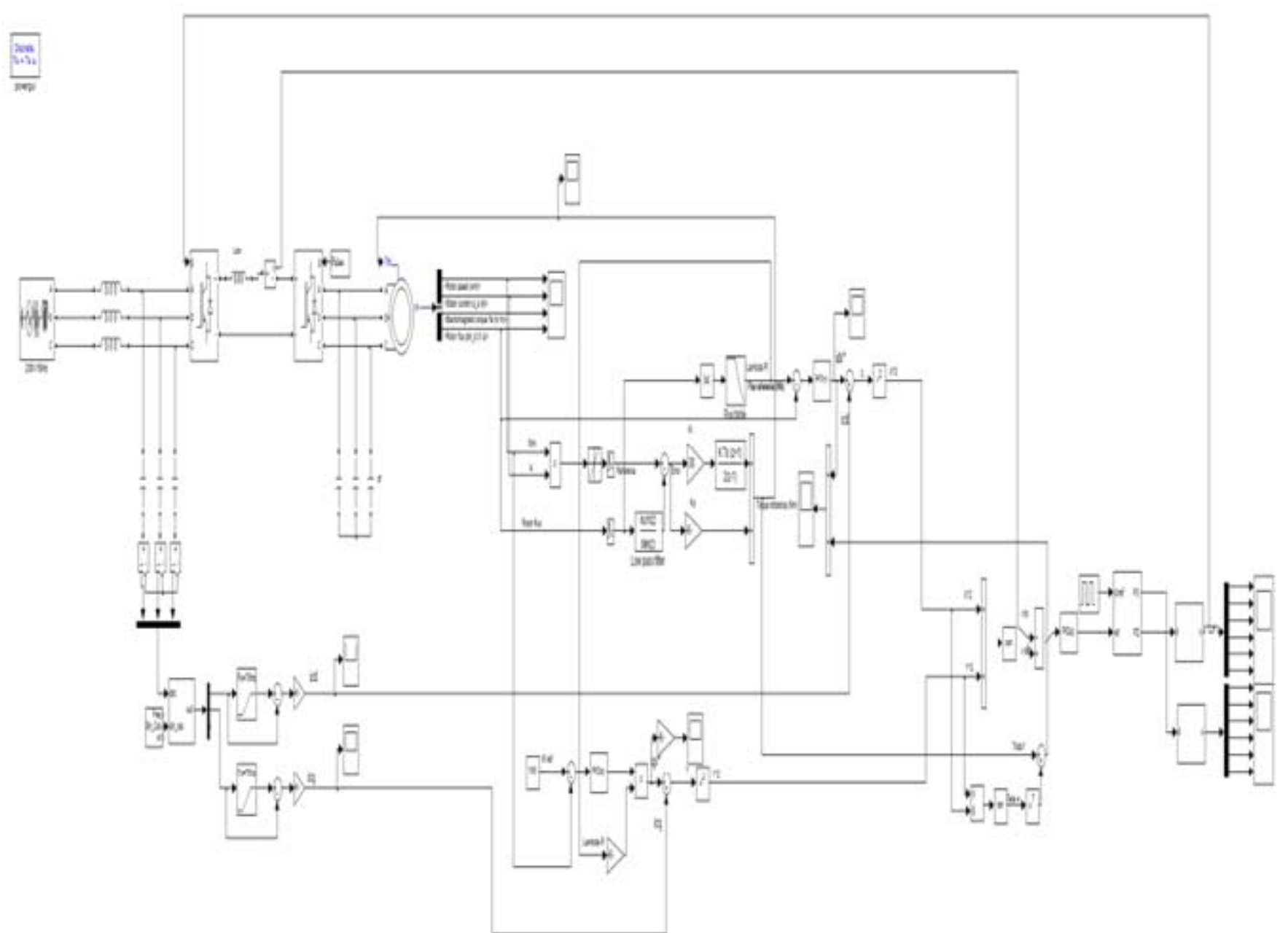

Figure 3: Simulink diagram CSI fed Induction Motor for Zero Speed Operation. 


\section{International Journal of Science and Research (IJSR) \\ ISSN (Online): 2319-7064}

Index Copernicus Value (2013): 6.14 | Impact Factor (2014): 5.611

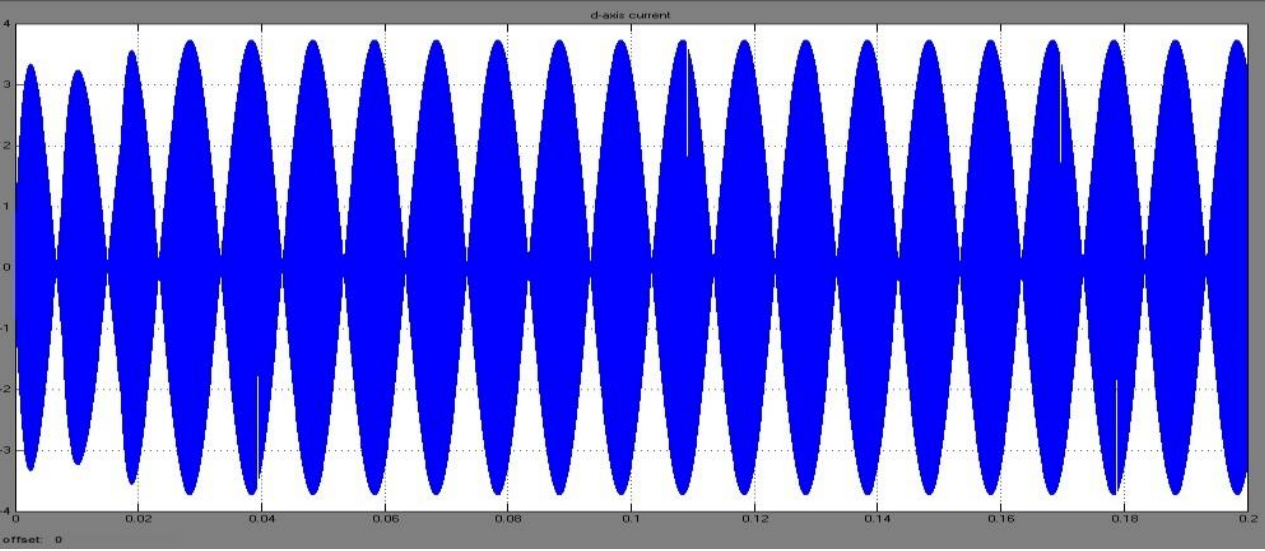

Figure 4: Resultant Input Waveform of d-axis

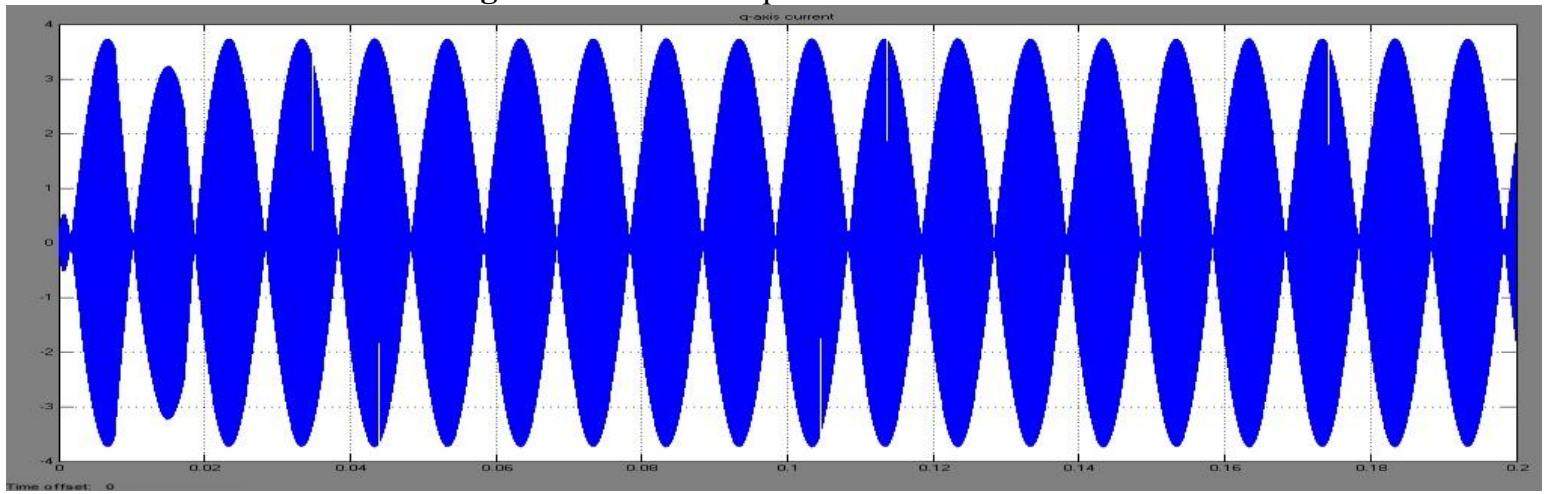

Figure 5: Resultant Input Waveform of q-axis

Resultant Output Waveform

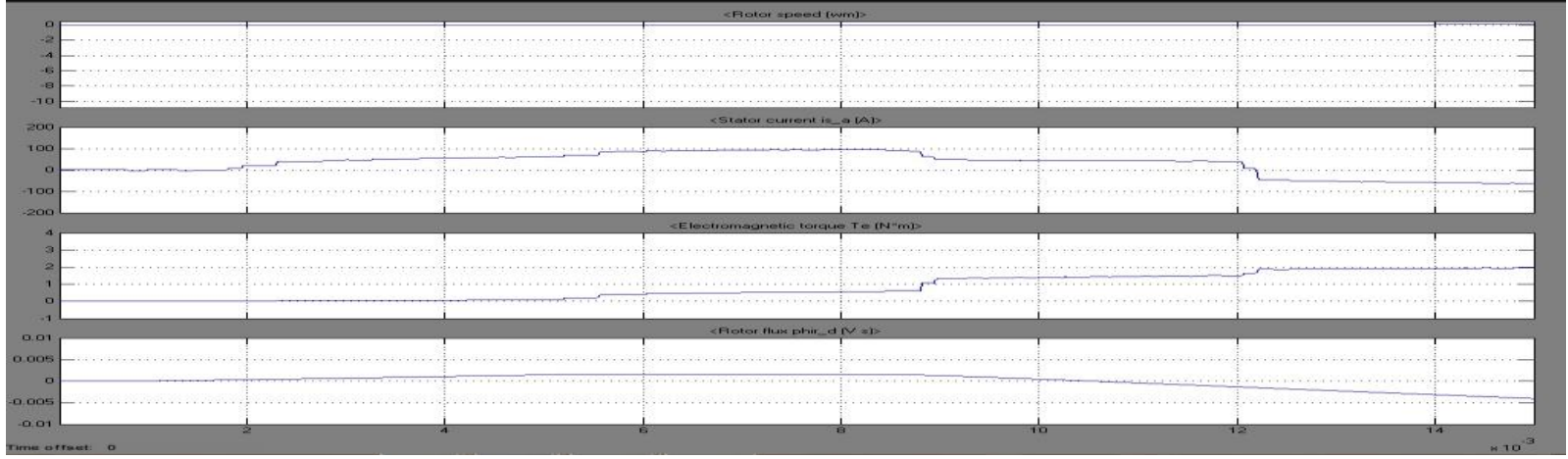

Figure 6: Output Waveform

The Figure.6.shows the resultant output waveform of the simulation diagram under CSI fed Induction Motor for zero speed operation. By employing the load torque feed forward control, the system performance under 0.5 p.u. step load torque is shown Figure.6 Due to the faster speed response, the dc-link current and stator $q$-axis current exhibit more transient resonances, while the motor speed drop is reduced to $8 \mathrm{r} / \mathrm{min}$. The speed recovery overshoot is increased as well, from 0 to $2.5 \mathrm{r} / \mathrm{min}$. In the meantime, the $q$-axis current exhibits a small amount of LF ripples after the implementation of the feed forward control, which is not presented in the simulation results. This is mainly introduced by the speed derivative, which highly depends on the encoder resolution and measurement noises. A comparatively large time constant $\tau$ is adopted for the lowpass filter to suppress such effect, which contributes to larger speed recovery overshoot.

\section{Conclusion}

Some of the strategies for sensor-less induction motor drives were reviewed and the effects of including a flux observer and a PWM instantaneous average measuring scheme were analyzed. When a speed sensor is available, the use of a flux observer showed to be an effective means to enable fast and precise flux estimation without any voltage-measuring scheme. The observer uses both voltage and current models. Since the current model depends on the speed, some additional mechanism must be used to ensure convergence for sensorless drives without some voltage measuring scheme. The implemented stator voltage-measuring scheme reduces the lower speed limit for induction motor drives with stator flux estimated only by the voltage model to be applied. The zero-speed operation of PWMCSI-fed IMD with speed senor is investigated. The Induction Motor used having the ratings of $0.25 \mathrm{HP}, 5 \mathrm{~A}, 415 \mathrm{~V}, 1400 \mathrm{RPM}$. The motor is controlled with rotor flux orientation, where the 


\section{International Journal of Science and Research (IJSR) \\ ISSN (Online): 2319-7064}

Index Copernicus Value (2013): 6.14 | Impact Factor (2014): 5.611

rotor flux is estimated using stator currents and motor speed. Due to the CSI-side filter capacitors, the stator currents are a portion of the inverter output currents. The influence of these capacitors on the motor control performance is systematically evaluated. A load torque observer with feed forward control is also utilized to improve the speed Dynamic response. Simulation and experiments show that the CSI-fed IMD works well at zero speed with promising speed dynamic performance.

\section{References}

[1] Bai Z, Ruan X, and Zhang Z, "A generic six-step direct PWM (SSDPWM) scheme for current source converter," IEEE Trans. Power Electron., vol. 25, no. 3, pp. 659666, Mar. 2010.

[2] Banerjee D and Ranganathan V.T, "Load-commutated SCR current source- inverter-fed induction motor drive with sinusoidal motor voltage and current," IEEE Trans. Power Electron., vol. 24, no. 4, pp. 1048-1061, Apr. 2009.

[3] Bierhoff M.H and Fuchs F.W, "Active damping for three-phase PWM rectifiers with high-order line-side filters," IEEE Trans. Ind. Electron., vol. 56, no. 2, pp. 371-379, Feb. 2009.

[4] Fangrui Liu, BinWu, Manish Pande and Navid Reza Zargari, "Zero-Speed Operation of High-Power PWM Current-Source-Inverter-Fed Induction Motor Drive," IEEE Transactions on power electronics, vol. 27, no. 6, June 2012.

[5] Hatua K and Ranganathan V.T, "A novel VSI and CSI fed dual stator induction motor drive topology for medium voltage drive applications," IEEE Trans. Power Electron., vol. 58, no. 8, pp. 3373-3382, Aug. 2011.

[6] Li R.T.H, Chung H.S, Lau W, and Zhou B, "Use of hybrid PWM and passive resonant snubber for a gridconnected CSI," IEEE Trans. Power Electron., vol. 25, no. 2, pp. 298-309, Feb. 2010.

[7] Li Y.M, Pande M, Zargari N.R, and Wu B, "Dc-link current minimization for high-power current-source motor drives," IEEE Trans. Power Electron., vol. 24, no. 1, pp. 232-240, Jan. 2009.

[8] Li Y.M, Pande M, Zargari N.R, and Wu B, "An input power factor control strategy for high-power currentsource induction motor drive with active front-end," IEEE Trans. Power Electron., vol. 25, no. 2, pp. 352359, Feb. 2010.

[9] Lops L. A.C and Naguib M F, "Space-vector-modulated hybrid bidirectional current source converter," IEEE Trans. Power Electron., vol. 25, no. 4, pp. 1055-1067, Apr. 2010 